\title{
Inversion of double-difference measurements from optical levelling for the Groningen gas field
}

\author{
P. A. Fokker and K. Van Thienen-Visser \\ TNO, Utrecht, the Netherlands \\ Correspondence to: P. A. Fokker (peter.fokker@tno.nl) \\ Published: 12 November 2015
}

\begin{abstract}
Hydrocarbon extraction lead to compaction of the gas reservoir which is visible as subsidence on the surface. Subsidence measurements can therefore be used to better estimate reservoir parameters. Total subsidence is derived from the result of the measurement of height differences between optical benchmarks. The procedure from optical height difference measurements to absolute subsidence is an inversion, and the result is often used as an input for consequent inversions on the reservoir. We have used the difference measurements directly to invert for compaction of the Groningen gas reservoir in the Netherlands. We have used a linear inversion exercise to update an already existing reservoir compaction model of the field. This procedure yielded areas of increased and decreased levels of compaction compared to the existing compaction model in agreement with observed discrepancies in porosity and aquifer activity.
\end{abstract}

\section{Introduction}

The Groningen gas field is a giant onshore field that has caused substantial subsidence since the start of its production in 1963. This subsidence has periodically been established by measuring the difference in height of stable benchmarks, using optical levelling. Pressures in the field have been closely monitored for reservoir management. History matching of the reservoir model on the observed pressures has resulted in a reasonably accurate pressure distribution development over the field.

There are a number of parameters in the relationship between the reservoir pressure and the subsidence which are more or less uncertain. The first one is the compaction coefficient, being dependent on the rock type and the porosity. There is also some uncertainty in the pressure estimates in some regions of the field, particularly in the connected aquifers, where pressure measurements are not available.

In the present paper we use the raw leveling difference measurements in conjunction with the prior knowledge about the Groningen gas reservoir in order to constrain the uncertainties. We employ an inverse algorithm to this end, but, instead of using interpreted heights, we use the originally measured height differences. In an earlier paper we reported the benefits this approach (Fokker and Van Thienen-Visser, 2015).

\section{Available data}

The Groningen gas field has been in production since 1963 . It is located onshore in the Northeast of the Netherlands. Extensive geological, geophysical and reservoir engineering data have been used to history-match the reservoir characteristics like geometry, porosity and permeability. We had access to the simulated pressure field at yearly dates from 1 January 1964 to 1 January 2017. The delta pressures were multiplied by the height and the estimated compaction coefficient for each grid cell, based on lithology, pressure depletion and porosity. For each $x-y$ location these numbers were accumulated over the reservoir layers in order to yield a prior estimate for the compaction grid at $9070 x-y$ locations for 54 times (Van Thienen-Visser et al., 2015). We remapped the provided compaction values to locations on a regular $400 \times 400 \mathrm{~m}^{2}$ grid for later manipulation. A map of the input compaction grid and the outline of the Groningen gas field in 2012 is provided in Fig. 1.

In the present study we focused on the use of data acquired through optical levelling. Usually, investigators use 


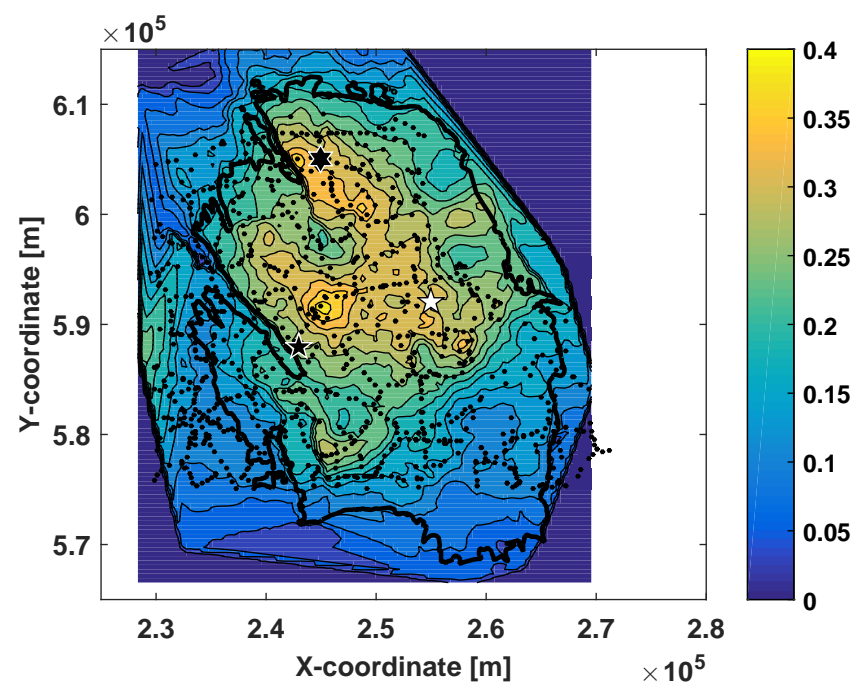

Figure 1. Prior estimate of the compaction field of the Groningen gas field in 2013 (color-coded), outline of the gas-bearing layers (solid line) and surface locations of the benchmarks used in the study (filled dots).

differences of the interpolated height maps to estimate surface movement. The procedure to obtain these differences includes the coupling to a reference benchmark or a set of reference benchmarks which are supposed to be stable, by integrating along the path of measurements to the stable benchmark. This procedure is sensitive to errors in the network and it accumulates the inaccuracy of all the measurements in the connecting path. The latter drawback can be addressed by providing the full covariance matrix of the resulting height estimates; this is, however, rarely done. Also reference benchmarks which, in hindsight, are not stable give rise to further inaccuracies. We have therefore chosen to use height difference measurements directly. The procedure to obtain double-difference estimates has been outlined in an earlier paper (Fokker and Van Thienen-Visser, 2015); it involves the determination of height differences between corresponding benchmark pairs in subsequent measurement campaigns, which have not necessarily been achieved in the same order.

Optical levelling campaigns have been performed many times in Groningen with different coverage. We had access to a total of 92 campaigns, dating from 1938 to 2012. Within a total of 7995 benchmarks, more than 26000 height differences had been measured. In this set, 1572 benchmarks had been identified as stable ones in the resulting optical levelling database. We have constructed differences between stable benchmarks only, using the measurement paths along the unstable ones, and used these to construct the double differences. Further, we discarded benchmarks west of the line with $x=230000 \mathrm{~m}$ and south of the line with $y=575000 \mathrm{~m}$ in the local coordinate system (RD) to exclude the influence of other sources of compaction in those areas (e.g. the deple- tion of the Annerveen gas field south of Groningen). Still, a total of 10860 double differences could be constructed between 987 benchmarks. The locations of these benchmarks are shown in Fig. 1.

\section{Forward model}

Gas production causes reservoir compaction, which, in turn, results in surface movement. Compaction in the reservoir may also change certain reservoir parameters. For the current study, a one-way coupling suffices - the change in porosity due to compaction only affects the reservoir pressure negligibly. We employed a linear-elastic model for the subsurface response, with the compacting blocks in the reservoir as source terms (Fokker and Orlic, 2006). Using an influence function approach, the subsidence at any surface point then is a superposition of the contributions of all compacting reservoir blocks. For the elastic profile in the subsurface we used a homogeneous elastic modulus down to a rigid basement at a depth of $5000 \mathrm{~m}$. The reservoir is located at a depth of $3000 \mathrm{~m}$. The connection to the double differences measured with the optical leveling can be made by making the appropriate time differences combined with space differences.

The goal of the present study was to employ an inverse algorithm on the interpreted double differences to improve the history match of the reservoir model and the predictive capability of the model in terms of reservoir pressures and subsidence. We considered the compaction of the reservoir as the uncertain parameter - the reservoir pressures and the porosities underlying it would involve too large computational efforts for this assessment. To map the uncertainty of the reservoir compaction we employed a field of multiplication factors at a spacing of $3200 \mathrm{~m}$ in space and 4 years in time. Values at the actual grid and intermediate times were obtained by interpolation. The prior multiplication values were defined as a constant value of unity over the field. A standard deviation of 0.3 was assumed. The mathematics of development

\section{Inverse model}

For the inverse model we define the vector $\boldsymbol{m}$ as the collection of adjustable model parameters, the vector $\boldsymbol{d}$ as the collection of double-difference data, and the matrix $\mathbf{G}$, working on the model parameters, as the forward model. The inverse problem is then formulated as the task of estimating the vector $\hat{\boldsymbol{m}}$ for which $\mathbf{G} \hat{\boldsymbol{m}}$ approaches the data vector $\boldsymbol{d}$ best. With additional information present in the form of a prior model $\left(\boldsymbol{m}_{0}\right)$ and covariance matrices of the measurements $\left(\mathbf{C}_{d}\right)$ and of the prior model $\left(\mathbf{C}_{m}\right)$, the conventional least-squares solution is obtained by maximizing the objective function $J$ given by Tarantola (2005) (or by minimizing $-\log [J]$ ): 


$$
\begin{aligned}
J= & \exp \left[-\frac{1}{2}\left(\boldsymbol{m}-\boldsymbol{m}_{0}\right)^{T} \mathbf{C}_{\boldsymbol{m}}^{-1}\left(\boldsymbol{m}-\boldsymbol{m}_{0}\right)-\frac{1}{2}\left(\boldsymbol{d}-\mathbf{G}_{\boldsymbol{m}}\right)^{T}\right. \\
& \left.\mathbf{C}_{\boldsymbol{d}}^{-1}\left(\boldsymbol{d}-\mathbf{G}_{\boldsymbol{m}}\right)\right]
\end{aligned}
$$

For the linear problem at hand, the estimate and its covariance are given by

$$
\begin{aligned}
\hat{\boldsymbol{m}}= & \boldsymbol{m}_{0}+\mathbf{C}_{\boldsymbol{m}} \mathbf{G}^{T}\left(\mathbf{G C}_{\boldsymbol{m}} \mathbf{G}^{T}+\mathbf{C}_{\boldsymbol{d}}\right)^{-1}\left(\boldsymbol{d}-\mathbf{G}_{\boldsymbol{m}_{0}}\right) \\
= & \boldsymbol{m}_{0}+\left(\mathbf{G}^{T} \mathbf{C}_{\boldsymbol{d}}^{-1} \mathbf{G}+\mathbf{C}_{\boldsymbol{m}}^{-1}\right)^{-1} \mathbf{G}^{T} \mathbf{C}_{\boldsymbol{d}}^{-1}\left(\boldsymbol{d}-\mathbf{G}_{\boldsymbol{m}_{0}}\right) \\
& \mathbf{C}_{\hat{\boldsymbol{m}}}=\mathbf{C}_{\boldsymbol{m}}-\mathbf{C}_{\boldsymbol{m}} \mathbf{G}^{T}\left(\mathbf{G} \mathbf{C}_{\boldsymbol{m}} \mathbf{G}^{T}+\mathbf{C}_{\boldsymbol{d}}\right)^{-1} \mathbf{G C}_{\boldsymbol{m}} \\
= & \left(\mathbf{G}^{T} \mathbf{C}_{\boldsymbol{d}}^{-1} \mathbf{G}+\mathbf{C}_{\boldsymbol{m}}^{-1}\right)^{-1}
\end{aligned}
$$

in which the first or second line of both expressions can be chosen according to the number of data points and model parameters (Tarantola, 2005). A smoothness constraint was added by extending the data vector with a number of elements equal to the number of multipliers in the model parameters, and by assigning the Laplacian working on $\boldsymbol{m}$ as the forward model for those elements. Furthermore, an independent constant vertical velocity for every benchmark was used as an additional unknown parameter to allow for movement not caused by the depletion of the gas field.

\section{Results}

The inversion exercise yielded an update of the fields of multiplication values and values for the autonomous movement of the benchmarks. With the original unit values and with the expected values of the multiplication factors, the forward model was rerun. The quality of the fit, indicated by $\chi^{2}=\frac{1}{N}\left(\mathbf{G}_{\boldsymbol{m}}-\boldsymbol{d}\right)^{2} / \sigma_{\boldsymbol{d}}^{2}$, improved from 8.8 to $5.9-$ the first and second number being calculated with the prior and the estimated model parameters, respectively. The remaining value around 6 , much larger than an optimal value around 1 , is presumably related to a remaining instability in the selected benchmarks, however it could also mean that the standard deviation of the height difference is too optimistic. The average of the background movement of the benchmark is zero; the standard deviation is $0.5 \mathrm{~mm}_{\text {year }}{ }^{-1}$.

There is a clear effect on the compaction fields. Examples of prior and updated compaction fields are given in Fig. 2. They show that around some areas the compaction levels must be adjusted to explain the measurements. These areas consistently return, independent of variations of the amount of smoothing or the precise form of the influence function in the forward model. More compaction than assumed in the prior model seems to have taken place around Ten Boer $\left[\left(x_{\mathrm{RD}} ; y_{\mathrm{RD}}\right)=(243000 ; 588000)\right]$; less around Delfzijl $\left[\left(x_{\mathrm{RD}} ; y_{\mathrm{RD}}\right)=(255000 ; 592000)\right.$ and less around Uithuizen $\left[\left(x_{\mathrm{RD}} ; y_{\mathrm{RD}}\right)=(245000 ; 605000)\right]$. The improvement of the double difference estimates and the effect on the subsidence estimates benchmarks is represented in Figs. 3 and 4.
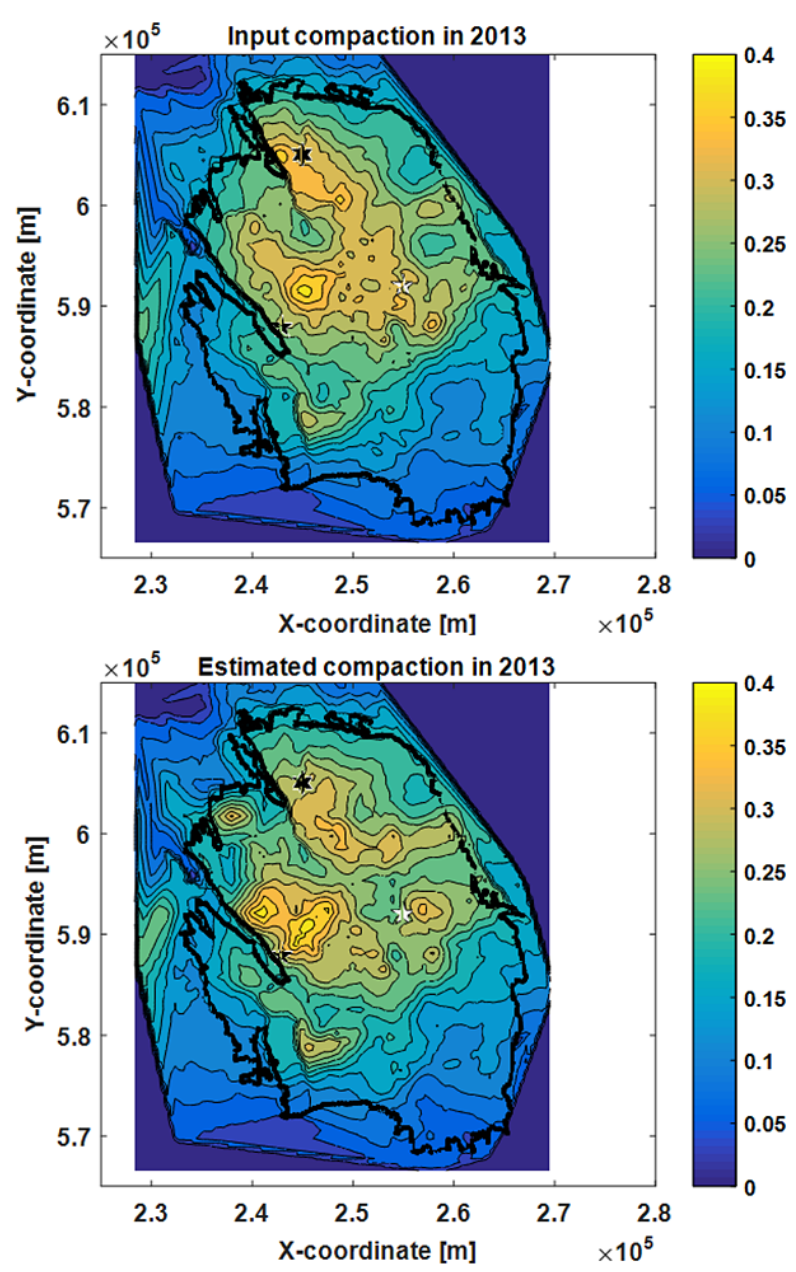

Figure 2. Prior compaction fields and estimated compaction fields in 2013.

\section{Discussion}

The correlation between measured and predicted double differences is much better for the estimated values of the multiplication factors than for the prior values. Still, the scatter remains considerable and there are many points with estimated value around zero that show comparatively large measured double differences. In view of this, it is remarkable that the inversion results in a consistent increase of compaction around Ten Boer and consistent decreases around Delfzijl and Uithuizen. This result was even apparent when no background movement was taken into account and the resulting correlation between measured and predicted double difference values was even worse. We assume that instabilities of individual benchmarks will cause deviations of double differences connected to them which are compensated with deviations with opposite sign for double differences starting from them.

Independent support for the updated compaction field has been found in a separate study (Van Thienen-Visser and Bre- 


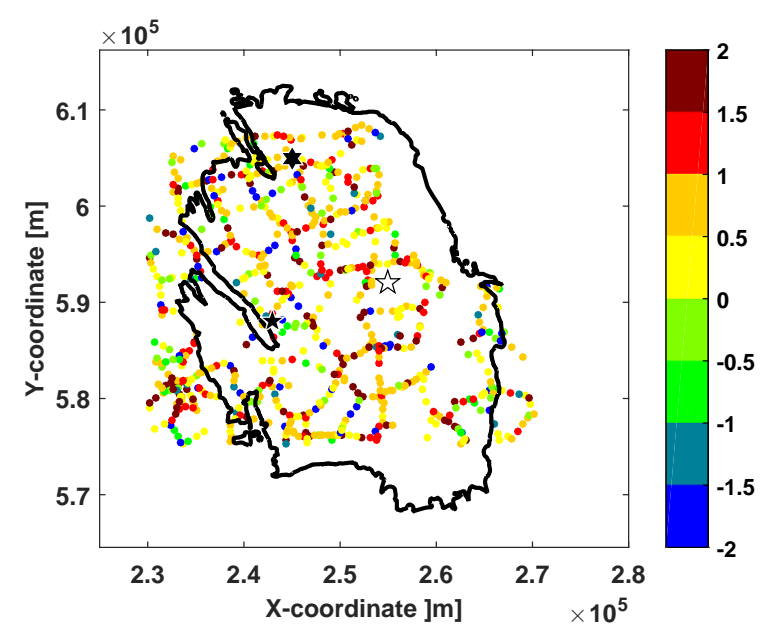

Figure 3. Improvement of the fit of double differences measured for two out of the 92 campaigns towards 1993 (starting times are variable for the different points). The color code indicates the ratio between prior and posterior offset: $\left(\mathbf{G}_{\boldsymbol{m}_{\mathbf{E}}}-\boldsymbol{d}\right) /\left(\mathbf{G}_{\boldsymbol{m}_{0}}-\boldsymbol{d}\right)$. Absolute values of this number smaller than 1 (yellow or green) indicate improvement.

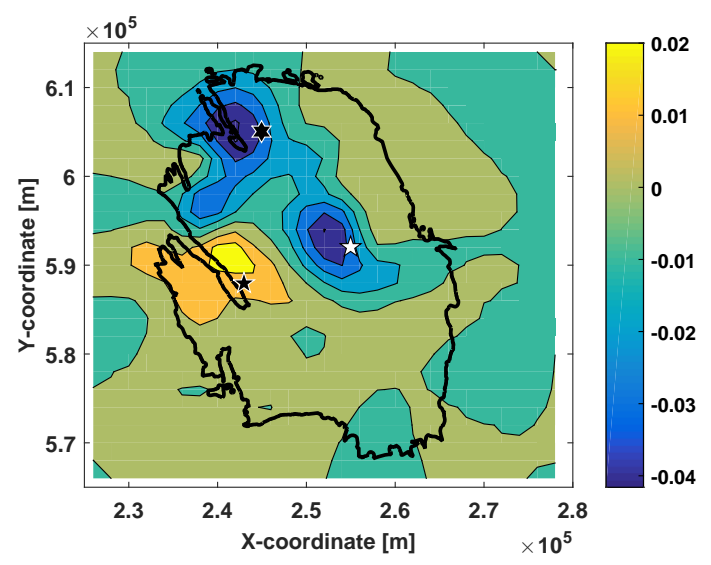

Figure 4. Effect of the compaction adjustment on the calculated subsidence in 2013.

unese, 2015). In that study, a different forward compaction model was employed and the predicted surface subsidence was compared to differences of interpreted heights at stable benchmarks and PS-InSAR measurement of the surface movement velocity. The areas that we found here were also identified in that study, and an additional effort was already recommended there to improve the subsurface model in those areas as it pointed towards inaccuracies of the porosity model and the assumed aquifer activity.

\section{Conclusions}

The present study proves the possibility of using double differences of optical levelling between stable benchmarks for the determination of reservoir parameters by its application on the Groningen gas field. The inverse study that we performed yielded a consistent update of the compaction of Groningen gas field during the lifetime of the field. The area around Ten Boer is compacting more than in the prior compaction model; the areas around Delfzijl and Uithuizen less. This is consistent with independent results obtained from comparing predicted subsidence with temporal differences of interpreted benchmark elevations. A renewed effort of reservoir modelling is required to improve the understanding of the reservoir in these areas.

Acknowledgements. The geological model and measured height differences were provided by NAM.

\section{References}

Fokker, P. A. and Orlic, B.: Semi-analytic modelling of subsidence, Math. Geol., 38 , 565-589, 2006.

Fokker, P. A. and Van Thienen-Visser, K.:. On the use of double differences in inversion of surface movement measurements, $\mathrm{Pa}$ per ARMA 15-096, presented at the 49th US Rock Mechanics/Geomechanics Symposium, San Francisco, CA, USA, 28 June-1 July 2015.

Tarantola, A.: Inverse Problem Theory and Methods for Model Parameter Estimation, SIAM, Paris, France, 64-68, 2005.

Van Thienen-Visser, K. and Breunese, J. N.: Induced seismicity of the Groningen gas field: history and recent developments, The Leading Edge, special issue Injection Induced Seismicity, 34, 664-671, 2015.

Van Thienen-Visser, K., Pruiksma, J., and Breunese, J. N.: Compaction and subsidence of the Groningen gas field in the Netherlands, Proc. IAHS, in this volume, 2015. 